\title{
Motivação acadêmica de estudantes de Medicina: uma análise na perspectiva da Teoria da Autodeterminação
}

Medical students' academic motivation: an analysis from the perspective of the Theory of Self-Determination

\author{
Anizio de Almeida Cadête Filho' (i) aniziocadete@gmail.com \\ José Maria Peixoto' ${ }^{1}$ (D) jmpeixoto.prof@gmail.com \\ Eliane Perlatto Moura' 1 (1) elianeperlatto@gmail.com
}

\section{RESUMO}

Introdução: Os princípios da Teoria da Autodeterminação são pertinentes para a educação profissional, na medida em que a diferenciação e expressão dos tipos primários de motivação têm implicações para os múltiplos desfechos da aprendizagem.

Objetivo: Este estudo teve como objetivos avaliar a motivação acadêmica em estudantes do quarto ano de Medicina em duas instituições de ensino e discutir os resultados na perspectiva da Teoria da Autodeterminação.

Método: Trata-se de estudo transversal e quantitativo conduzido por questionário autorrespondido contendo 18 questões sociodemográficas e a Escala de Motivação Acadêmica (EMA). A amostra foi constituída por 147 estudantes do quarto ano de Medicina de escolas com metodologias de ensino distintas: 73 estudantes da instituição A (aprendizagem baseada em problemas - ABP) e 74 estudantes da instituição B (tradicional). Conduziu-se uma análise univariada em que se utilizaram os testes t de Student, a Análise de Variância (ANOVA), o teste qui-quadrado e a correlação de Pearson. Posteriormente, fez-se uma análise de regressão linear múltipla.

Resultados: Observou-se um perfil moderado de motivação intrínseca (MI) e extrínseca (ME) na amostra de estudantes, com médias de MI superiores às da ME. Níveis elevados de MI foram observados nos domínios de motivação para realização e para o saber, sendo o prazer de ampliar conhecimentos e o aumento da competência profissional os fatores que mais impactaram positivamente a motivação. Na Ml, o fator com menor pontuação foi a universidade como local de prazer. A ME foi relacionada ao desejo de ter uma boa vida no futuro, incluindo remuneração. Observou-se que as variáveis sexo feminino, idade menor que 23 anos, morar sozinho, ter feito o curso todo na mesma instituição e a escola com metodologia ABP influenciaram de forma positiva na motivação.

Conclusão: Os estudantes demonstraram níveis de $\mathrm{MI}$ superiores à da ME, com menor pontuação atribuída à universidade como local de prazer. Esse fato apresenta uma oportunidade para estudos futuros, que poderão verificar os fatores do ambiente escolar que se relacionam à motivação em aprender dos estudantes. A ME foi associada às expectativas futuras de vida. Conhecer as variáveis que caracterizam a autorregulação da aprendizagem é fundamental para embasar estratégias de ensino que contribuam para a melhoria do processo de ensino-aprendizagem.

Palavras-chave: Motivação; Aprendizagem; Educação Médica; Estudantes de Medicina.

\section{ABSTRACT}

Introduction: The principles of the Theory of Self-Determination are relevant to professional education, as the differentiation and expression of the primary types of motivation have implications for the multiple learning outcomes.

Objective: To evaluate academic motivation in 4th-year medical students at two teaching institutions and discuss the results from the perspective of the Theory of Self-Determination.

Method: The present is a cross-sectional and quantitative study, conducted through a self-answered questionnaire containing 18 sociodemographic questions and the Academic Motivation Scale (AMS). The sample consisted of 147 4th-year medical students from schools with different teaching methodologies: 73 students from Institution A (Problem-based learning - PBL) and 74 students from Institution B (traditional methodology). A univariate analysis was conducted using Student's t test, Analysis of Variance (ANOVA), chi-square test and Pearson's correlation, followed by Multiple Linear Regression analysis.

Results: There was a moderate profile of intrinsic (IM) and extrinsic (EM) motivation in the sample of students, with a higher IM than EM average. High levels of IM were observed in the domains of motivation for achievement and for knowledge, with the satisfaction of increasing one's knowledge and increasing professional competence being the factors that most positively impacted motivation. In IM, the factor with the lowest score was the university as a place of enjoyment. EM was related to the desire of having a good life in the future, including monetary compensation. It was observed that the variables female gender, age under 23 years, living alone, having attended the entire course at the same institution and school with the PBL methodology had a positive influence on motivation.

Conclusion: The students demonstrated higher levels of IM than those of EM, with a lower score attributed to the university as a place of enjoyment. This fact shows an opportunity for future studies, which will be able to verify the factors of the school environment related to the students' motivation to learn. EM was associated with future life expectations. To know the variables that characterize the self-regulation of learning is crucial to support teaching strategies that contribute to the improvement of the teaching-learning process.

Keywords: Motivation; Learning; Medical Education; Medical Students.

${ }^{1}$ Universidade José do Rosário Vellano, Belo Horizonte, Minas Gerais, Brasil.

Editora-chefe: Daniela Chiesa

| Editor associado: Maurício Abreu Pinto Peixoto

Recebido em 11/05/20; Aceito em 23/03/21. | Avaliado pelo processo de double blind review. 


\section{INTRODUÇÃO}

A motivação é um componente fundamental para o processo de desempenho humano ${ }^{1-3}$. Sua correlação com os contextos de ensino e aprendizagem tem despertado interesse da literatura, uma vez que apresenta poder preditivo no âmbito escolar, pois um estudante motivado é capaz de transformar o conhecimento adquirido em incentivo para aprendizagem continuada 4 . Percepções objetivas ou subjetivas sobre o nível de eficácia pessoal ou competência no ambiente de estudos acadêmicos podem afetar a intensidade ou a orientação da motivação ${ }^{5}$.

A motivação pode ser definida como uma necessidade ou um desejo vinculado à vontade de cumprir um objetivo esperado $^{6}$, ou como o comportamento regulado por necessidade e instinto para atingir um determinado resultado ${ }^{7}$. Segundo Pintrich ${ }^{8,9}$, no olhar acadêmico, a motivação para a realização de uma tarefa pode ser estimulada por três componentes: 1. uma razão ou um propósito para realizá-la; 2. a crença do indivíduo em sua capacidade para executá-la; e 3. as reações afetivas obtidas com o desempenho da atividade.

Dois tipos de motivação podem ser caracterizados: a motivação intrínseca, em que se faz algo pelo interesse e prazer inerentes à ação; e a motivação extrínseca, em que se faz algo por causa de consequência ou desfecho distinto da ação. A motivação extrínseca assume diferentes formas, que se distinguem pelo grau de internalização de valores e regulação de condutas, refletindo diferentes níveis de autonomia. Uma motivação autônoma relaciona-se com mais qualidade no aprendizado, maior persistência e melhor ajuste psicológico dos aprendizes, em qualquer estágio de formação ou escolarização ${ }^{10,11}$. Ou seja, comportamentos extrinsecamente motivados são governados pela relação entre as perspectivas de ganhos e perdas instrumentais (por exemplo, incentivos), enquanto comportamentos intrinsecamente motivados são dirigidos pelo bem próprio (por exemplo, o prazer da tarefa).

\section{Procurando compreender os determinantes} motivacionais e as formas autodeterminadas de motivação, Deci et al. ${ }^{12}$ propuseram o que chamam de Teoria da Autodeterminação, que faz distinções entre os diferentes níveis de motivação: a intrínseca, a extrínseca e a desmotivação. A Teoria da Autodeterminação enfoca, em particular, como internalizamos ideias, valores, objetivos e intenções sob a influência de contextos sociais incorporados, como a interação do indivíduo com o ambiente e o contexto ${ }^{13}$, e tem inspirado diversos estudos na área de educação no Brasil|14-17.

O princípio de que o aprendizado se estabelece a partir de mecanismos de motivação se liga às bases da Teoria da Autodeterminação. Esta, por sua vez, compreende o comportamento humano como sendo incentivado por três necessidades psicológicas primárias e universais fundamentais para que a autodeterminação se desenvolva:

- Autonomia: reflete o desejo de participar de atividades em que a possibilidade de escolha esteja presente.

- Capacidade: está ligada ao sentir-se capacitado e confiante para realizar um determinado comportamento com determinada aptidão.

- Relação social: trata-se da necessidade de perceber que o comportamento é reconhecido positivamente por outras pessoas ou que a sua prática facilita a socialização ${ }^{13}$ (Figura 1).

Os princípios da Teoria da Autodeterminação são pertinentes para a educação profissional, na medida em que a diferenciação e expressão dos tipos primários de motivação têm implicações para os múltiplos desfechos da aprendizagem. Assim, as atribuições de causalidade ${ }^{18,19}$ e os conceitos ligados não só ao estabelecimento e ao alcance de metas ${ }^{20,21}$, mas também ao tipo de motivação (intrínseca ou extrínseca) ou às orientações motivacionais ${ }^{12}$, constituem-se importantes tópicos a serem investigados.

A linha de estudos motivacionais relacionados ao aprendizado universitário e, mais especificamente, médico tem ganhado destaque. Hayat et al. ${ }^{22}$ observaram correlação positiva entre a motivação intrínseca e a extrínseca no desempenho acadêmico de estudantes de Medicina da Shiraz University of Medical Sciences. Kusurkar et al. ${ }^{23}$, ao estudarem o perfil motivacional de estudantes de Medicina do sexto ano da University Medical Center Utrecht, verificaram correlação positiva entre motivação intrínseca e adequadas estratégias de estudo, horas de autoestudo e desempenho acadêmico e correlação negativa com exaustão. Sobral ${ }^{24}$ constatou padrões distintos de motivação intrínseca e motivação extrínseca (também denominadas autônomas e controladas,

Figura 1. Necessidades incentivadoras do comportamento pela Teoria da Autodeterminação.

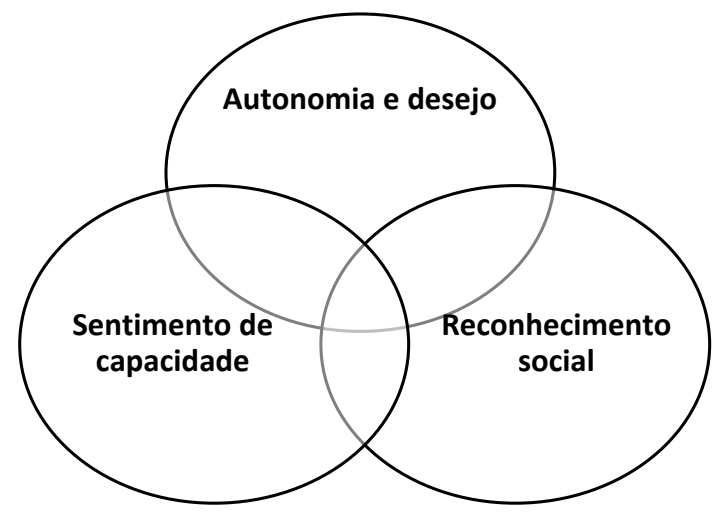


respectivamente) que parecem estar relacionados com a percepção dos estudantes de Medicina sobre o aprendizado e o ambiente educacional.

Segundo Lozano et al. ${ }^{25}$, em um modelo dinâmico motivacional, um "aluno motivado" considera as atividades educativas propostas úteis ou interessantes (percepção do valor de uma atividade educacional), sente-se capaz de concluir as atividades para sua própria satisfação (autoeficácia percebida) e tem a impressão de ser responsável pelo progresso de seu aprendizado (percepção de controlabilidade).

Considerando a importância do construto motivação para o processo de aprendizagem no ensino médico e sua relação com a Teoria da Autodeterminação, este estudo objetivou avaliar a motivação acadêmica em estudantes do quarto ano de Medicina em duas instituições de ensino de Belo Horizonte, em Minas Gerais, e discutir os resultados na perspectiva da Teoria da Autodeterminação. Os resultados deste estudo poderão servir de parâmetros para a elaboração de intervenções que possam impactar a aprendizagem dos estudantes de Medicina.

\section{MÉTODO}

Trata-se de um estudo transversal e quantitativo conduzido por meio de um questionário autorrespondido para avaliar a motivaçã o dos estudantes de Medicina. A amostra foi composta por 147 estudantes do quarto ano de Medicina de duas instituições particulares de ensino situadas em Belo Horizonte, em Minas Gerais, com metodologias de ensino diferentes, sendo 73 discentes da instituição A (metodologia de aprendizado baseado em problemas - ABP) e 74 da instituição B (metodologia tradicional). Essa amostra representou, respectivamente, $44,2 \%$ e $55,2 \%$ do total de estudantes de Medicina matriculados no quarto ano das instituições $A$ e $B$. $O$ quarto ano de ensino foi escolhido por tratar-se do último período em que os dois cursos apresentam diferenças em sua metodologia de ensino. A partir desse momento, inicia-se o internato, quando as atividades de ensino passam a apresentar maior homogeneidade em ambas as instituições, uma vez que se caracterizam por atividades práticas em serviços de saúde. Até esse momento do curso, as duas instituições apresentam diferenças nas metodologias. Na tradicional, a transição do ensino dos temas das áreas básicas para as práticas é mais definida, quando essa metodologia é comparada à proposição do currículo orientado pelo $A B P$, em que essas atividades ocorrem simultaneamente. A amostra foi determinada por conveniência entre os estudantes que cursavam o quarto ano e estavam presentes em sala de aula no momento de aplicação do questionário. O convite foi realizado pelos pesquisadores nas salas de aula, em horário predeterminado com o professor, seguido do preenchimento do questionário pelos estudantes que concordaram em participar e assinaram o termo de consentimento. $O$ instrumento de pesquisa continha 46 questões: 18 referentes à avaliação sociodemográfica, para identificação de fatores comportamentais, de saúde, familiares e relacionados ao ambiente de ensino, elaboradas pelos pesquisadores; e 28 concernentes à Escala de Motivação Acadêmica (EMA) elaborada por Vallerand et al. ${ }^{26}$ e validada para o português por Sobral ${ }^{24}$. Optou-se por essa escala devido ao grande número de citações dela na literatura. O tempo para preenchimento foi de livre necessidade dos estudantes, mas apresentou duração média de 20 minutos.

- Questionário sociodemográfico: Constituído por 18 questões sociodemográficas, acadêmicas e familiares. As variáveis analisadas foram: nome, gênero, idade, renda familiar, ter parente de primeiro grau médico, estado civil, morar sozinho ou com a família, ter trabalhado remunerado, número de horas semanais trabalhadas, ter algum problema de saúde, usar medicação antidepressiva, usar medicação crônica, ter feito o curso todo na instituição, estar regularmente matriculado no curso, exercer atividade extracurricular e motivo principal da atividade extracurricular.

- Escala de Motivação Acadêmica (EMA): A versão original da escala foi desenvolvida na língua francesa do Canadá por Vallerand et al.26, pressupondo a multifatorialidade dos processos motivacionais, e traduzida e adaptada para o português do Brasil por Sobral24. Écomposta por 28 itens e pontuada em uma escala do tipo Likert de sete pontos ( 1 = nenhuma correspondência e $7=$ total correspondência). A escala apresenta sete dimensões, com o propósito de mensurar três tipos de motivação intrínseca (MI) e três tipos de motivação extrínseca (ME), além da desmotivação (quadros 1, 2 e 3).

Os tipos de motivação intrínseca abrangem: motivação intrínseca para saber (fazer algo pelo prazer que decorre de aprender, explorar ou entender), motivação intrínseca para realizar coisas (fazer algo pelo prazer que decorre da busca de realização ou criação de coisas) e motivação intrínseca para vivenciar estímulo (fazer algo a fim de experimentar sensações estimulantes, de natureza sensorial ou estética). Os tipos de motivação extrínseca incluem: regulação por identificação (fazer algo porque se decidiu fazê-lo), regulação por introjeção (fazer algo porque se pressiona a si próprio a fazê-lo) e regulação externa (fazer algo porque se sente pressionado por outros a fazê-lo). Por fim, o conceito de desmotivação implica 
ausência de percepção de contingências entre as ações e seus desfechos (falta de motivos intrínsecos ou extrínsecos). A análise dos tipos de motivação seguiu os parâmetros descritos por Lopes et al. ${ }^{14}$ (quadros 1, 2 e 3).

Os itens apresentados nos quadros 1, 2 e 3 foram analisados pelas médias dos escores obtidos por meio da escala de Likert com pontuação de 1 a 7. Sendo assim, adaptados do estudo de Azevedo et al. ${ }^{27}$, utilizaram-se os seguintes pontos de corte: $\leq 3,0$ (baixa motivação), > 3,0 e < 6,0 (média motivação) e $\geq 6,0$ (alta motivação).
Mediu-se o desempenho acadêmico pela média geral das notas obtidas, levando-se em consideração todas as matérias cursadas até o momento do estudo, que foram disponibilizadas pelas instituições.

Utilizaram-se a média do somatório dos escores dos 28 itens da EMA para avaliar a motivação acadêmica em cada instituição (escore total) e a média dos escores dos itens relacionados a cada uma das dimensões para avaliar a motivação dos alunos em cada domínio do instrumento. Para a análise dos dados, realizou-se estatística descritiva e conduziu-se uma

Quadro 1. Motivação intrínseca para

\begin{tabular}{ccl}
\hline & - & porque sinto satisfação e prazer enquanto aprendo coisas novas. \\
saber & - & pelo prazer quando descubro coisas novas que nunca tinha visto ou conhecido antes. \\
& - & pelo prazer que tenho em ampliar meu conhecimento sobre assuntos que me atraem. \\
& - & porque meus estudos permitem que continue a aprender sobre muitas coisas que me interessam. \\
\hline realização & porque acho que a formação universitária ajuda a me preparar melhor para a carreira que escolhi. \\
& - & porque o curso me capacitará, no final, a entrar no mercado de trabalho de uma área de que eu gosto. \\
& - & porque eu creio que a formação universitária aumentará minha competência como profissional. \\
\hline & - & porque gosto muito de vir à universidade. \\
vivenciar estímulos & porque para mim a universidade é um prazer. \\
& - pelo prazer que tenho quando me envolvo em debates com professores interessantes. \\
& pela euforia que sinto quando leio sobre vários assuntos interessantes.
\end{tabular}

Quadro 2.

Motivação extrínseca por

\begin{tabular}{ccl}
\hline & - & pelo prazer que sinto quando supero a mim mesmo no estudo. \\
identificação & - & pelo prazer que sinto quando supero a mim mesmo em algumas de minhas realizações pessoais. \\
& pela satisfação que sinto quando estou no processo de realização de atividades acadêmicas difíceis. & porque a universidade me permite sentir uma satisfação pessoal na minha busca por excelência \\
& na formação.
\end{tabular}

\section{Quadro 3. Desmotivação.}

- Honestamente, não sei; acho que estou perdendo meu tempo na universidade.

- Já tive boas razões para isso; agora, entretanto, eu me pergunto se devo continuar.

- Não atino [percebo] por que venho à universidade e, francamente, não me preocupo com isso.

- Não sei; não entendo o que estou fazendo na universidade. 
análise univariada em que se utilizaram os testes $t$ de Student e/ou Análise de Variância (ANOVA), o teste qui-quadrado e a correlação de Pearson com o propósito de pré-selecionar as variáveis de interesse para a condução da análise multivariada, em que foi aplicada a análise de regressão linear múltipla. $\mathrm{O}$ nível de significância utilizado foi de $5 \%(p<0,05)$. Utilizou-se o programa SPSS 14.0 for Windows (software estatístico).

O projeto foi encaminhado ao Comitê de Ética em Pesquisa da Universidade José do Rosário Vellano, tendo recebido parecer favorável, com o Certificado de Apresentação para Apreciação Ética (CAAE) n 84589418.5.0000.5143.

\section{RESULTADOS}

O estudo envolveu 147 estudantes que cursavam o quarto ano de Medicina de duas instituições de ensino situadas em Belo Horizonte. No geral, estas eram as características dos participantes: a maioria do gênero feminino (70\%); faixa etária de 19 a 30 anos; predomínio de renda familiar maior que dez mil reais (63\%); não ter parente médico (69\%); estar solteiro (96\%), não ter trabalho remunerado (97\%); não ter problema de saúde (80\%); não usar medicamento antidepressivo (82\%) ou para doença crônica (82\%); não ser fumante (97\%); não ter mudado de instituição de ensino no curso de Medicina (85\%); exercer atividade extracurricular (82\%); e estar regular no curso (94\%).

A análise dos escores da motivação acadêmica dos 147 estudantes demonstrou valor médio de escore de motivação intrínseca maior que o observado na motivação extrínseca (Tabela 1). Na avaliação individual dos itens que compõem a EMA, em relação à motivação intrínseca, observou-se que o domínio "motivação intrínseca para realização" obteve a maior média de escore. Os itens que receberam maior pontuação

Tabela 1. Escores médios de motivação dos estudantes de Medicina do quarto ano avaliada pela Escala de Motivação Acadêmica (EMA), 2018.

\begin{tabular}{ccc}
\hline Domínios & $\begin{array}{c}\text { Escore } \\
\text { (média /dp) }\end{array}$ & $\mathbf{p}^{*}$ \\
\hline Motivação intrínseca (MI) & $5,9 \pm 0,7^{*}$ & $<0,001$ \\
MI para saber & $6,0 \pm 1,1$ & \\
MI para realização & $6,3 \pm 0,9$ & \\
MI para vivenciar estímulos & $4,5 \pm 1,4$ & \\
Motivação extrínseca (ME) & $5,3 \pm 1,2 *$ & \\
ME para identificação & $5,0 \pm 1,6$ & \\
ME para introjeção & $4,1 \pm 1,7$ & \\
ME para controle externo & $5,7 \pm 1,2$ & \\
Desmotivação & $1,6 \pm 1,1^{*}$ & \\
\hline
\end{tabular}

Base de dados: 147 alunos. A probabilidade de significância refere-se ao teste $t$ de Student para amostras pareadas $\left(p^{*}\right)$. foram os relacionados à percepção de que a formação universitária prepara melhor para a carreira que escolheu e aumenta a competência profissional. $\mathrm{O}$ item que recebeu a menor pontuação foi sobre a influência da universidade na escolha da orientação profissional. No domínio "motivação intrínseca para saber", o item mais pontuado foi o prazer em ampliar conhecimentos sobre assuntos atrativos; e o menos pontuado, o prazer em descobrir coisas novas. O domínio "motivação intrínseca para vivenciar estímulos" foi o que apresentou a menor média de escore. Nesse domínio, o item relacionado à leitura de assuntos interessantes foi o mais pontuado, enquanto o item que considera a universidade um prazer apresentou pontuação menor.

Em relação à motivação extrínseca, o domínio "motivação extrínseca para controle externo" obteve a maior média de escore, com as maiores pontuações para os itens referentes a ter uma vida boa e uma boa remuneração no futuro, ao passo que a obtenção de um emprego de prestígio recebeu a menor pontuação. No domínio"motivação extrínseca para identificação”, o item mais pontuado foi o prazer de se superar nas realizações pessoais; e o menos pontuado, a satisfação com as realizações de atividades acadêmicas difíceis. O domínio "motivação extrínseca para introjeção" obteve a menor média. O item mais pontuado foi referente a mostrar a si mesmo que pode ser bem-sucedido nos estudos; e o menos pontuado, mostrar a si mesmo que é um indivíduo inteligente. No domínio "desmotivação", o item mais pontuado foi não ver razão para continuar fazendo o curso.

Neste estudo, os fatores sociodemográficos, acadêmicos e familiares que influenciaram nos escores de motivação obtidos pela EMA foram: gênero, idade, morar sozinho, tomar medicamento antidepressivo, não ter feito o curso todo na mesma instituição e a metodologia de ensino adotada pela instituição (Tabela 2). O gênero feminino apresentou médias de escores mais elevadas na maioria dos domínios das escalas de motivação $(p<0,05)$, com exceção da motivação extrínseca para controle externo e desmotivação, em que as médias de escores se apresentaram semelhantes entre os sexos $(p>0,05)$. Foi identificada uma correlação negativa significativa e fraca $(r$ $=-0,19-p<0,029)$ entre as idades na subescala de motivação extrínseca para controle externo, na qual os estudantes mais jovens tendem a apresentar resultados superiores. Os estudantes que moram sozinho apresentaram resultados superiores $(p<0,05)$ nas subescalas motivação intrínseca para saber e para vivenciar estímulo, e motivação extrínseca para identificação quando comparados com os alunos que moram com a família. Os estudantes que fazem uso de medicamento antidepressivo apresentaram valores superiores $(p<0,05)$ na subescala que avalia desmotivação. Os estudantes da instituição com metodologia ABP apresentaram valores mais 
Tabela 2. Influência dos fatores sociodemográficos, familiares e acadêmicos na média de escore de motivação medida pela Escala de Motivação Acadêmica (EMA) de estudantes de Medicina - Belo Horizonte, 2018.

\begin{tabular}{|c|c|c|c|c|c|c|c|c|c|c|c|c|c|c|}
\hline \multirow{3}{*}{ Variáveis } & \multicolumn{14}{|c|}{ Domínios } \\
\hline & \multirow{2}{*}{$\begin{array}{c}\begin{array}{c}\text { MI } \\
\text { saber }\end{array} \\
\text { média } \pm \mathrm{dp} \\
\end{array}$} & \multirow[t]{2}{*}{$p$} & \multirow{2}{*}{$\begin{array}{c}\text { MI } \\
\text { realização } \\
\text { média } \pm d p\end{array}$} & \multirow[t]{2}{*}{$p$} & \multirow{2}{*}{$\begin{array}{c}\text { MI } \\
\text { vivenciar } \\
\text { estímulo } \\
\text { média } \pm \text { dp }\end{array}$} & \multirow[t]{2}{*}{$\mathbf{p}$} & \multirow{2}{*}{$\begin{array}{c}\text { ME } \\
\text { identificação } \\
\text { média } \pm \text { dp }\end{array}$} & \multirow[t]{2}{*}{$p$} & \multirow{2}{*}{$\begin{array}{c}\text { ME } \\
\text { introjeção } \\
\text { média } \pm d p\end{array}$} & \multirow[t]{2}{*}{$p$} & \multirow{2}{*}{$\begin{array}{c}\text { ME } \\
\begin{array}{c}\text { controle } \\
\text { externo }\end{array} \\
\text { média } \pm \mathrm{dp}\end{array}$} & \multirow[t]{2}{*}{ p } & \multirow{2}{*}{$\begin{array}{l}\text { Desmotivação } \\
\text { média } \pm \text { dp }\end{array}$} & \multirow[t]{2}{*}{$p$} \\
\hline & & & & & & & & & & & & & & \\
\hline \multicolumn{15}{|l|}{ Sexo } \\
\hline Feminino & $5,4 \pm 1,3$ & 0,001 & $5,8 \pm 1,1$ & 0,002 & $3,8 \pm 1,3$ & $<0,001$ & $4,2 \pm 1,6$ & $<0,001$ & $3,6 \pm 1,9$ & 0,031 & $5,6 \pm 1,4$ & 0,511 & $1,9 \pm 1,2$ & 0,073 \\
\hline Masculino & $6,2 \pm 1,0$ & & $6,4 \pm 0,8$ & & $4,8 \pm 1,3$ & & $5,3 \pm 1,5$ & & $4,3 \pm 1,6$ & & $5,7 \pm 1,1$ & & $1,5 \pm 1,0$ & \\
\hline \multicolumn{15}{|l|}{ Idade } \\
\hline$r$ & 0,07 & 0,424 & $-0,09$ & 0,270 & 0,05 & 0,584 & $-0,02$ & $0,0,73$ & $-0,13$ & 0,144 & $-0,19$ & 0,029 & $-0,05$ & 0,598 \\
\hline \multicolumn{15}{|c|}{ Renda familiar (R\$) } \\
\hline $\begin{array}{c}\text { Até } \\
5.000,00\end{array}$ & $6,5 \pm 0,4$ & 0,394 & $6,5 \pm 0,6$ & 0,773 & $4,9 \pm 1,5$ & 0,407 & $5,3 \pm 1,3$ & 0,212 & $4,8 \pm 1,1$ & 0,210 & $6,3 \pm 0,7$ & 0,391 & $1,5 \pm 0,6$ & 0,469 \\
\hline $\begin{array}{c}\text { Acima de } \\
5.000,00\end{array}$ & $6,2 \pm 0,8$ & & $6,4 \pm 0,7$ & & $4,8 \pm 1,3$ & & $4,8 \pm 1,6$ & & $4,1 \pm 1,5$ & & $5,6 \pm 1,4$ & & $1,5 \pm 1,0$ & \\
\hline \multicolumn{15}{|c|}{ Parente médico } \\
\hline Sim & $5,8 \pm 1,3$ & 0,306 & $6,1 \pm 1,0$ & 0,312 & $4,3 \pm 1,3$ & 0,113 & $4,8 \pm 1,7$ & 0,337 & $4,3 \pm 1,7$ & 0,408 & $5,7 \pm 1,4$ & 0,943 & $1,8 \pm 1,2$ & 0,303 \\
\hline Não & $6,1 \pm 1,1$ & & $6,3 \pm 0,9$ & & $4,6 \pm 1,4$ & & $5,1 \pm 1,5$ & & $4,0 \pm 1,7$ & & $5,7 \pm 1,1$ & & $1,6 \pm 1,0$ & \\
\hline \multicolumn{15}{|c|}{ Morar sozinho } \\
\hline Sim & $6,2 \pm 1,0$ & 0,029 & $6,4 \pm 0,8$ & 0,056 & $4,8 \pm 1,3$ & 0,010 & $5,4 \pm 1,5$ & 0,006 & $4,3 \pm 1,6$ & 0,130 & $5,7 \pm 1,2$ & 0,684 & $1,5 \pm 0,8$ & 0,059 \\
\hline Não & $5,8 \pm 1,3$ & & $6,1 \pm 1,0$ & & $4,3 \pm 1,4$ & & $4,6 \pm 1,6$ & & $3,9 \pm 1,7$ & & $5,7 \pm 1,2$ & & $1,8 \pm 1,3$ & \\
\hline \multicolumn{15}{|c|}{ Problema de saúde } \\
\hline Sim & $6,0 \pm 1,1$ & 0,748 & $6,1 \pm 0,7$ & 0,353 & $4,9 \pm 1,2$ & 0,047 & $5,2 \pm 1,6$ & 0,412 & $4,5 \pm 1,7$ & 0,211 & $5,8 \pm 1,0$ & 0,577 & $1,7 \pm 0,8$ & 0,790 \\
\hline Não & $6,0 \pm 1,2$ & & $6,3 \pm 1,0$ & & $4,4 \pm 1,4$ & & $4,9 \pm 1,6$ & & $4,0 \pm 1,7$ & & $5,7 \pm 1,2$ & & $1,6 \pm 1,1$ & \\
\hline \multicolumn{15}{|c|}{ Medicamento antidepressivo } \\
\hline Sim & $5,7 \pm 1,4$ & 0,291 & $5,9 \pm 1,0$ & 0,065 & $4,4 \pm 1,6$ & 0,575 & $4,6 \pm 1,8$ & 0,193 & $4,0 \pm 1,6$ & 0,725 & $5,8 \pm 1,1$ & 0,798 & $2,1 \pm 1,1$ & 0,012 \\
\hline Não & $6,0 \pm 1,1$ & & $6,3 \pm 0,9$ & & $4,6 \pm 1,3$ & & $5,1 \pm 1,5$ & & $4,1 \pm 1,7$ & & $5,7 \pm 1,2$ & & $1,5 \pm 1,0$ & \\
\hline \multicolumn{15}{|c|}{ Medicamento para doença crônica } \\
\hline Sim & $5,9 \pm 1,1$ & 0,792 & $6,2 \pm 0,6$ & 0,574 & $4,9 \pm 1,2$ & 0,110 & $5,0 \pm 1,6$ & 0,899 & $4,4 \pm 1,7$ & 0,284 & $5,9 \pm 0,9$ & 0,291 & $1,9 \pm 1,1$ & 0,182 \\
\hline Não & $6,0 \pm 1,2$ & & $6,3 \pm 1,0$ & & $4,4 \pm 1,4$ & & $5,0 \pm 1,6$ & & $4,0 \pm 1,7$ & & $5,7 \pm 1,2$ & & $1,6 \pm 1,1$ & \\
\hline \multicolumn{15}{|c|}{ Curso todo na instituição } \\
\hline Sim & $6,0 \pm 1,1$ & 0,692 & $6,3 \pm 0,9$ & 0,335 & $4,5 \pm 1,3$ & 0,877 & $4,9 \pm 1,6$ & 0,778 & $4,3 \pm 1,7$ & 0,011 & $5,7 \pm 1,2$ & 0,937 & $1,6 \pm 1,0$ & 0,739 \\
\hline Não & $6,1 \pm 1,4$ & & $6,1 \pm 1,1$ & & $4,6 \pm 1,6$ & & $5,1 \pm 1,7$ & & $3,3 \pm 1,5$ & & $5,7 \vee 1,2$ & & $1,7 \pm 1,4$ & \\
\hline Atividade e & tracurricular & & & & & & & & & & & & & \\
\hline Sim & $6,0 \pm 1,1$ & 0,588 & $6,3 \pm 0,9$ & 0,751 & $4,5 \pm 1,4$ & 0,909 & $5,0 \pm 1,6$ & 0,346 & $4,1 \pm 1,7$ & 0,588 & $5,8 \pm 1,1$ & 0,142 & $1,6 \pm 1,0$ & 0,337 \\
\hline Não & $5,9 \pm 1,2$ & & $6,2 \pm 0,9$ & & $4,5 \pm 1,5$ & & $4,7 \pm 1,7$ & & $4,3 \pm 1,6$ & & $5,3 \pm 1,4$ & & $1,9 \pm 1,4$ & \\
\hline Desempent & o escolar & & & & & & & & & & & & & \\
\hline$r$ & 0,02 & 0,847 & $-0,03$ & 0,695 & 0,05 & 0,566 & $-0,03$ & 0,703 & $-0,16$ & 0,052 & $-0,08$ & 0,338 & $-0,1$ & 0,206 \\
\hline
\end{tabular}

Base de dados: 147 alunos. MI: motivação intrínseca; ME: motivação extrínseca. A probabilidade de significância refere-se ao teste t de Student, Análise de Variância e análise de correlação.

elevados de motivação intrínseca e motivação extrínseca em todos os domínios da escala, exceto na motivação extrínseca para controle externo que foi semelhante nas duas instituições. Ressalta-se que o domínio desmotivação teve escore mais elevado na instituição com metodologia tradicional.

\section{DISCUSSÃO}

Este estudo teve como objetivos avaliar a motivação acadêmica de estudantes do quarto ano do curso de Medicina em duas instituições de ensino de Belo Horizonte e discutir os resultados na perspectiva da Teoria da Autodeterminação. Os resultados obtidos pela EMA revelaram níveis moderados de motivação intrínseca e motivação extrínseca, sendo as médias da motivação intrínseca superiores às da extrínseca. Ressalta-se que níveis elevados de motivação intrínseca foram observados nos domínios de motivação para realização e para o saber, sendo o prazer de ampliar conhecimentos sobre temas atrativos e o aumento da competência profissional 
os fatores que mais impactaram positivamente a motivação dos estudantes analisados. Entretanto, escores baixos foram observados no item frequentar a universidade por prazer. A motivação extrínseca se relacionou com o desejo de ter uma boa vida e uma boa remuneração no futuro.

O predomínio da motivação intrínseca revela que o que mais motiva os estudantes é o prazer pela atividade em si. Os dados obtidos no presente estudo estão em consonância com os resultados de pesquisas anteriores no que concerne ao predomínio da motivação intrínseca em estudantes universitários tanto do curso de Medicina ${ }^{15}$ quanto de outros cursos das áreas biológicas, humanas e exatas ${ }^{14,28,29}$.

$O$ fato de a motivação intrínseca predominar no grupo de alunos estudados é um dado esperado e positivo em ambas as instituições que fizeram parte deste estudo, pois, de acordo com a Teoria da Autodeterminação, os seres humanos são naturalmente inclinados para o desenvolvimento pessoal, procurando internalizar elementos psíquicos e sociais que favoreçam a construção de um sentido sobre si mesmos30, sendo a motivação intrínseca o fenômeno que melhor representa o potencial positivo da natureza humana, configurando-se como uma tendência natural para exercitar as próprias capacidades, buscando novidades e desafios.

Refere-se ao envolvimento em determinada atividade por sua própria causa, por esta ser interessante, envolvente ou, de alguma forma, geradora de satisfação, o que promove maior interesse dos estudantes pela aprendizagem e a confiança nas próprias capacidades e atributos ${ }^{16,31}$. Assim, a motivação intrínseca representa o melhor tipo de motivação, pois é autônoma ou autodeterminada. A curiosidade dos alunos e o interesse pessoal pelo material de aprendizagem direcionam para um aprendizado caracterizado pelo senso de liberdade psicológica e um lócus interno de causalidade ${ }^{31}$, o que pode ser demonstrado neste estudo pelas elevadas médias de escore observadas nos domínios da motivação intrínseca para realizações (que se refere à interação com o meio e o sentimento de competência para criar) e no domínio motivação para saber (que está relacionado com a exploração, curiosidade e objetivos). A motivação intrínseca conduz à criação de metas internas, nomeadas como metas de aprendizagem ${ }^{32}$, diferentemente das metas externas ou de rendimento que guiam a motivação extrínseca, que objetiva o recebimento de recompensas ou ausência de punição ${ }^{32}$. Estudantes orientados por metas de aprendizagem buscam mais o desenvolvimento de suas competências, enquanto aqueles orientados por metas de rendimento procuram a obtenção de avaliações positivas ${ }^{32}$.

Um dado interessante observado neste estudo é que, dentre os componentes da motivação intrínseca, aquele que atingiu a menor pontuação na autopercepção da motivação dos estudantes foi o item que relaciona a universidade a local de prazer. A literatura aponta diversos fatores relacionados ao ambiente escolar capazes de influenciar a motivação para aprendizagem, dos quais o mais importante é o que ocorre em sala de aula, desde os materiais didáticos utilizados, a metodologia de ensino empregada até os fatores afetivos vigentes na relação professor-aluno ${ }^{33,34}$. A sala de aula deve ser um local afável, que propicia ao aluno um sentimento de pertencimento, sendo a motivação mediada pelo professor, pelo ambiente da sala e pela cultura escolar ${ }^{31}$.

Uma vez que a motivação é influenciada pela interação dos componentes intrínsecos e extrínsecos ${ }^{34}$, a possibilidade de despertar a motivação do aluno pela universidade, relacionando-a como um local de prazer, é uma importante contribuição identificada neste estudo, que salienta a oportunidade para que professores e gestores possam planejar ações metodológicas, de capacitação e do próprio ambiente educacional, que oportunizem vivências estimuladoras para os estudantes.

Apesar da predominância de motivação intrínseca nos estudantes avaliados, o componente extrínseco, regulado por recompensas e restrições, também se mostrou presente. Segundo Boruchovitch ${ }^{28}$ e Ryan et al. ${ }^{33}$, a presença da orientação extrínseca, relacionada com comportamentos que são utilizados como um meio para atingir um fim e não por si mesmos, quando acompanhada da motivação intrínseca, pode também produzir bons resultados na aprendizagem.

No domínio da motivação extrínseca, as maiores pontuações foram observadas nos itens referentes às expectativas futuras de vida, incluindo remuneração, e naqueles relacionados ao prazer de se superar para realizações pessoais; e verificaram-se as menores pontuações em atividades acadêmicas difíceis e obtenção de um emprego de prestígio. Observa-se, portanto, que os itens que mais mobilizam a motivação extrínseca são os relacionados à satisfação de desejos pessoais e à obtenção de autonomia, e aqueles que despertam o interesse do estudante mais por identificação do que pela obrigação em fazer. Afinal, a motivação humana é guiada pela necessidade de autorrealização, que considera o atendimento das necessidades básicas, como a segurança e a estima ${ }^{35}$. Nossos dados demonstraram que os estudantes reconhecem a universidade como o melhor meio de preparação para a carreira escolhida, o que confirma a proposição de que a necessidade da formação acadêmica seja reflexo do desejo de se desenvolver como indivíduo ${ }^{35}$.

É importante salientar que, apesar de o componente extrínseco da motivação ser relevante para a aprendizagem, ele deve ser utilizado com o objetivo de despertar a motivação intrínseca, que é associada a alto grau de desempenho educacional e prazer dos alunos ${ }^{36}$. O uso de recompensas 
extrínsecas sem planejamento pode promover redução na motivação intrínseca, uma vez que não desperta no estudante a satisfação em aprender por aprender ${ }^{37}$.

O conhecimento dos objetivos e das orientações motivacionais dos estudantes são importantes, pois fundamentam a sequência motivacional desencadeada diante de uma tarefa a ser realizada, com impacto na aprendizagem ${ }^{28}$. O tipo de orientação motivacional dos estudantes tem sido associado a diferenças nas variáveis gênero, idade, semestre, características do estabelecimento de ensino, crenças, percepção de tempo futuro, entre outras ${ }^{28}$. Orsini et al. ${ }^{38}$ conduziram uma revisão sistemática de fatores que influenciam a motivação e os categorizaram em cinco grupos: 1. determinantes intrapessoais, como idade e sexo; 2 . determinantes interpessoais, como condições acadêmicas; 3. resultados cognitivos, como crenças; 4. resultados afetivos, como ansiedade ou depressão; e 5. resultados comportamentais, como o envolvimento acadêmico. A escolha, o reconhecimento de sentimentos e a oportunidade de autodireção também aumentaram a motivação intrínseca, porque esses fatores facilitaram um maior senso de autonomia ${ }^{39}$. Assim, a análise da influência das variáveis sociodemográficas dos estudantes é importante, na medida em que elas têm implicações quer no seu processo acadêmico e social de integração, quer no seu percurso acadêmico e profissional em curto, médio e longo prazos ${ }^{40}$.

Em conformidade com o que foi encontrado por outros pesquisadores ${ }^{16,24,41,42}$, os resultados do presente estudo demonstraram que as estudantes do sexo feminino apresentaram médias de escore mais elevadas de motivação, quando comparadas aos estudantes do sexo masculino. Em relação à idade, observou-se que os estudantes mais jovens apresentaram escore maior de motivação extrínseca, o que não corrobora os achados da literatura, uma vez que alguns estudos não encontraram associação entre idade e motivação, enquanto em outros os alunos mais velhos exibiram um perfil mais autônomo ${ }^{43-45}$. Diferentemente dos resultados obtidos por Azevedo et al. ${ }^{27}$, observou-se que os estudantes que moram sem os pais apresentaram níveis mais elevados de motivação. $\mathrm{Na}$ tentativa de explicar esse fato, podemos inferir que a nova realidade social vivenciada pelo estudante que se encontra longe de sua família estimula o desenvolvimento da autonomia que é uma das bases da Teoria da Autodeterminação. Outro achado importante foi a ausência de vínculo entre o desempenho acadêmico e o grau de motivação, o que reforça a motivação autodeterminada dos estudantes avaliados.

Por fim, observou-se que os alunos da escola que adota a metodologia do APB apresentaram médias dos escores da EMA maiores em relação aos da escola com metodologia tradicional. Esse fato pode ser explicado à luz da Teoria da
Autodeterminação, que estabelece como pilares da motivação a autonomia, a competência e o relacionamento ${ }^{10}$, que são aspectos favorecidos pela metodologia $\mathrm{APB}^{39,46}$. Na concepção desenvolvida por Ryan e Deci ${ }^{33}$, o ambiente acadêmico é preponderante, uma vez que ele pode facilitar ou inibir a motivação intrínseca, em decorrência do suporte ou da obstrução das necessidades psicológicas de competência e autonomia dos aprendizes. Segundo Reeve ${ }^{47}$, estudantes que se percebem autônomos em suas interações escolares apresentam resultados positivos em relação à motivação, ao engajamento, ao desenvolvimento, à aprendizagem, à melhoria do desempenho e ao estado psicológico. Assim, um contexto que estimula a autonomia é propício à aprendizagem e ao desempenho com efeito na motivação, na internalização de valores e regras, que proporcionarão às pessoas maior autodeterminação. No entanto, não é possível afirmar, a partir desses dados, que a metodologia do APB seria capaz de isoladamente influenciar a motivação dos estudantes, uma vez que este estudo não objetivou comparar as duas instituições de ensino em relação às diversas variáveis que se associam à motivação dos discentes.

Apesar da tendência natural para o desenvolvimento pessoal que os estudantes possuem, é importante observar que fatores externos podem dificultar que a motivação intrínseca se manifeste em sua plenitude ${ }^{30}$. Com o objetivo de favorecer a motivação intrínseca, o ensino deveria estimular a autonomia dos estudantes de Medicina, ter o planejamento das atividades partindo das necessidades deles, com desafios oportunos ao nível cognitivo em que se encontram, incentivando a participação ativa e o desenvolvimento de responsabilidade pelo próprio aprendizado, além de fornecer apoio emocional e feedback construtivo ${ }^{48}$.

Sabendo que a motivação se constitui de estados dinâmicos e não traços estáveis de personalidade ${ }^{49,50}$, como limitações do estudo, podemos citar:

- A metodologia transversal impede que os resultados possam ser generalizados. Por conta disso, estudos longitudinais se fazem necessários para melhor conhecimento do perfil de motivação dos estudantes ao longo do curso, bem como da influência dos fatores sociodemográficos nesse percurso.

- O uso de uma escala de autorrelato traz o risco da subjetividade das respostas pelo efeito da desejabilidade social.

- Esta pesquisa não avaliou os fatores do ambiente escolar que se relacionam à motivação do estudante, que poderão ser explorados em estudos futuros.

- O estudo não comparou as instituições de ensino 
com o objetivo de verificar se a metodologia de ensino empregada se relaciona à motivação dos estudantes. Em razão disso, estudos futuros poderão avançar nesse conhecimento.

Este estudo contribui para fomentar a discussão da necessidade de se considerar, além dos elementos cognitivos curriculares, um dos mais importantes aspectos relacionados à aprendizagem: a motivação. A motivação se relaciona ao aprendizado profundo e favorece o hábito da aprendizagem ao longo da vida profissional, proporcionando a formação de profissionais competentes. Considerando que no ambiente acadêmico a motivação e a aprendizagem são integralmente relacionadas, é importante que o tema seja considerado por gestores e professores durante o planejamento das atividades acadêmicas, inclusive quando da organização do currículo, que deveriam levar em conta estratégias para o atendimento das necessidades de autonomia dos estudantes, de modo a promover o sentimento de confiança em suas competências e relacionamentos sociais, que são as necessidades básicas defendidas pela Teoria da Autodeterminação ${ }^{51}$.

\section{CONCLUSÃO}

Este estudo evidenciou que os estudantes de Medicina do quarto ano apresentaram um perfil moderado de motivação intrínseca e motivação extrínseca, com médias da motivação intrínseca superiores às da extrínseca. Os níveis elevados de motivação intrínseca foram observados nos domínios de motivação para realização e para o saber, sendo o prazer de ampliar conhecimentos e o aumento da competência profissional os fatores que mais impactaram positivamente a motivação dos estudantes. Dentre os fatores de motivação intrínseca, aquele que recebeu a menor pontuação foi a universidade como local de prazer. Esse fato apresenta uma oportunidade para estudos futuros, que poderão verificar os fatores do ambiente escolar que se relacionam à motivação em aprender dos estudantes. A motivação extrínseca estava altamente relacionada com o desejo de ter uma boa vida no futuro, incluindo remuneração. A motivação acadêmica foi influenciada pelos fatores: sexo feminino, morar sozinho e estudar em instituição com metodologia APB. Conhecer as variáveis que caracterizam a autorregularão da aprendizagem é fundamental para embasar estratégias de ensino que contribuam para a melhoria do processo de ensino-aprendizagem.

\section{CONTRIBUIÇÃO DOS AUTORES}

Anizio de Almeida Cadête Filho participou ativamente do delineamento da pesquisa, da coleta de dados, da discussão dos resultados e da redação do artigo. Trata-se dos resultados da dissertação de mestrado. José Maria Peixoto participou da revisão e aprovação da versão final do texto. Eliane Perlatto Moura participou ativamente do delineamento da pesquisa, da coleta de dados, da discussão dos resultados, da redação do artigo, da revisão e aprovação da versão final do texto.

\section{CONFLITO DE INTERESSES}

Os autores declaram não haver conflito de interesses neste estudo.

\section{FINANCIAMENTO}

Declaramos que não houve financiamento para a realização desta pesquisa.

\section{REFERÊNCIAS}

1. Campbell JP, Pritchard RD. Motivation theory in industrial and organizational psychology. In: Dunnette MD, Hough L, editors. Handbook of industrial and organizational psychology. Chicago: Rand McNally College; 1976. p. 63-130.

2. Maier NRF. Psychology in industry. 2nd ed. Boston: Houghton Mifflin; 1955.

3. Pinder WCC. Work motivation in organizational behavior. 2nd ed. New York: Psychology Press; 2011.

4. Spinath B. Development and modification of motivation and selfregulation in school contexts: introduction to the special issue. Learn Instr 2005;15(2):85-6.

5. Ryan RM, Deci EL. Intrinsic and extrinsic motivations: classic definitions and new directions. Contemp Educ Psychol. 2000;25(1):54-67.

6. Todorov JC, Moreira MB. O conceito de motivação na psicologia. Rev Bras Ter Comport Gogn. 2005;7(1):119-32.

7. Deese J. Principles of psychology. Boston: Allyn and Bacon, 1964.

8. Pintrich PR. Editor's comment. Educ Psychol. 1991;26(3-4):199-205.

9. Pintrich PR. The role of goal orientation in self-regulated learning. In: Boekaerts M, Pintrich PR, Zeidner M. Handbook of self-regulation. Cambridge: Academic Press; 2000. p. 451-502.

10. Deci EL, Vallerand RJ, Pelletier LG, Ryan RM. Motivation and education: the self-determination perspective. Educ Psychol. 1991;26(3-4):325-46.

11. Deci EL, Ryan RM, Williams GC. Need satisfaction and the self-regulation of learning. Learn Individ Differ. 1996;8(3):165-83.

12. Deci EL, Ryan RM. The general causality orientations scale: selfdetermination in personality. J Res Pers. 1985;19(2):109-34.

13. Deci EL, Ryan RM. Motivation, personality, and development within embedded social contexts: an overview of self-determination theory. In: Deci EL, Ryan RM. The Oxford handbook of human motivation. Rochester: University of Rochester; 2012. p. 85-107.

14. Lopes LMS, Pinheiro FMG, Silva ACR, Abreu ES. Aspectos da motivação intrínseca e extrínseca: uma análise com discentes de ciências contábeis da Bahia na perspectiva da teoria da autodeterminação. RGFC. 2015;5(1):21-39.

15. Sobral DT. Motivação do aprendiz de medicina: uso da escala de motivação acadêmica. Psicol Teor Pesqui. 2003;19(1):25-31.

16. Guimarães SER, Bzuneck JA, Boruchovitch E. Estilos motivacionais de professores: propriedades psicométricas de um instrumento de avaliação. Psicol Teor Pesqui. 2003;19(1):17-24.

17. Guimarães SEF, Boruchovitch E. O estilo motivacional do professor e a motivação intrínseca dos estudantes: uma perspectiva da teoria da autodeterminação. Piscol Reflex Crit. 2004;17(2):143-50.

18. Weiner, B. A theory of motivation for some classroom experiences. J Educ Psychol. 1979;71(1):3-25.

19. Weiner, B. Transforming cultural plurality into theoretical unity. In Mcinerney MD, Van Etten, S, editors. Big theories revisited. Greenwich Information Age; 2004. p. 13-29. 
20. Nicholls JG. Achievement motivation: conceptions of ability, subjective experience, task choice, and performance. Psychol Rev. 1984;91(3):328-46.

21. Elliot ES, Dweck CS. Goals: an approach to motivation and achievement. J Pers Soc Psychol. 1988;54(1):5-12.

22. Hayat AA, Salehi A, Kojuri J. Medical student's academic performance: the role of academic emotions and motivation. J Adv Med Educ Prof. 2018;6(4):168-75.

23. Kusurkar RA, Croiset G, Galindo-Garré F, Ten Cate O. Motivational profiles of medical students: association with study effort, academic performance and exhaustion. BMC Med Educ. 2013;13(1):87-95.

24. Sobral DT What kind of motivation drives medical students' learning quests? Med Educ. 2004;38(9):950-7.

25. Lozano AB, Mascarenhas SA, Castro FV, Rioboo AP. Motivação académica e atribuições causais: a escala QEAP48 numa amostra de alunos universitários de Rondônia e Humaitá (Amazónia, Brasil). Actas do X Congresso internacional Galeco-Português de Psicopedagogia. Braga: Universidade do Minho; 2009 p. 4497-508.

26. Vallerand RJ, Pelletier L, Blais MR, Brière NM. The academic motivation scale: a measure of intrinsic extrinsic and amotivation in education. Educ Pshycol Measu 1992;52(4):1003-17.

27. Azevedo PTÁCC, Caminha MDF, Andrade CRSD, Godoy CGD, Monteiro RLS, Falbo AR. Motivação Intrínseca do Estudante de Medicina de uma faculdade com metodologia ativa no Brasil: estudo transversal. Rev Bras Educ Med. 2019;43(1):12-23.

28. Boruchovitch E. A motivação para aprender de estudantes em curso de formação de professores. Educ 2008;31(1):30-8.

29. Joly MCRA, Prates EAR. Avaliação da escala de motivação acadêmica em estudantes paulistas: propriedades psicométricas. Psico USF. 2011;16(2):175-84

30. Ten Cate TJ, Kusurkar RA, Williams GC. How self-determination theory can assist our understanding of the teaching and learning processes in medical education. Med Teach. 2011;33(12):961-73.

31. Vansteenkiste M, Sierens E, Soenens B, Luyckx K, Lens W. Motivational profiles from a self-determination perspective: the quality of motivation matters. J Educ Psychol. 2009;101(3):671-88.

32. Ribeiro F. Motivação e aprendizagem em contexto escolar. Profforma. 2011;03:1-5 [access in 26 apr 2020]. Available from: http://www.cefopna. edu.pt/revista/revista_03/pdf_03/es_05_03.pdf.2:

33. Ryan RM, Deci EL. Self-determination theory and the facilitation of intrinsic motivation, social development, and well-being. Am Psychol. 2000;55(1):68-78.

34. Lourenço $\mathrm{AA}$, de Paiva MOA. A motivação escolar e o processo de aprendizagem. Ciência \& Cognição. 2010;15(2):132-41.

35. Kusurkar RA, Croiset G, Mann KV, Custers E, Ten Cat O. Have motivation theories guided the development and reform of medical education curricula? A review of the literature. Med Acad. 2012;87(6):735-43.
36. Deci EL, Ryan RM. The general causality orientations scale: selfdetermination in personality. J Res Pers. 1985;19(2):109-34.

37. Lepper MR, Greene D, Nisbett RE. Minando o interesse intrínseco das crianças com recompensa extrínseca: um teste da hipótese de "superjustificação". J Pers Soc Psychol. 1973;28(1):129-37.

38. Orsini C, Binnie VI, Wilson SL. Determinants and outcomes of motivation in health professions education: a systematic review based on selfdetermination theory. J Educ Eval Health Prof. 2016;2:13-9.

39. Peixoto JM, Ribeiro MMF, Amaral CFS. Atitude do estudante de medicina a respeito da relação médico-paciente x modelo pedagógico. Rev Bras Educ Med. 2011;35(2)229-36.

40. Hojat $M$, Zuckerman $M$. Personality and specialty interest in medical students. Med Teach. 2008;30(4):400-6.

41. CaiY, Reeve J, Robinson DT. Home schooling and teaching style: comparing the motivating styles of home school and public school teachers. J Educ Psychol. 2002;94(2):372-80.

42. Zenorini RPC, Santos, AAA. A motivação e a utilização de estratégias de aprendizagem em universitários. Estudante universitário: característica e experiências de formação. Taubaté: Cabral Editora e Livraria Universitária; 2003.

43. Williams GC, Deci EL. Internalization of biopsychosocial values by medical students: a test of self-determination theory. J Pers Soc Psychol. 1996;70(4):767-79.

44. Tanaka M, Mizuno K, Fukuda S, Tajima S, Watanabe Y. Personality traits associated with intrinsic academic motivation in medical students. Med Educ. 2009;43:384-7.

45. Kusurkar R, Croiset G, ten Cate O. Implications of gender differences in motivation among medical students. Med Teach. 2013;35(2):173-4.

46. Toledo Júnior ACC, Ibiapina CDC, Lopes SCF, Rodrigues ACP, Soares SMS Aprendizagem baseada em problemas: uma nova referência para a construção do currículo médico. Rev Méd. Minas Gerais. 2008;18(2)123-31.

47. Reeve J. Teachers as facilitators: what autonomy-supportive teachers do and why their students benefit. Elem Sch J. 2006;106(3):225-37.

48. Kusurkar RA, ten Cate TJ, Vos CMP, Westers P, Croiset G. How motivation affects academic performance: a structural equation modelling analysis. Adv Health Sci Educ. 2013;18(1):57-69.

49. Murphy PK, Alexander PAA. Motivated exploration of motivation terminology. Contemp Educ Psychol. 2000;25(1):3-53.

50. Zimmerman BJ. Self-efficacy: an essential motive to learn. Contemp Educ Psychol. 2000;25(1):82-91.

51. Kusurkar RA, Croiset G, ten Cate OTJ. Twelve tips to stimulate intrinsic motivation in students through autonomy-supportive classroom teaching derived from self-determination theory. Med Teach. 2011;33(12):978-982. 